\title{
Case Report 304
}

Robert H. Jongeward, Jr., M.D., Ph.D. ${ }^{1}$, William Martel, M.D. ${ }^{1 *}$, Dean S. Louis, M.D. ${ }^{2}$, Matthias I. Okoye, M.D. ${ }^{3}$, and Norman Walter, M.D. ${ }^{4}$

Departments of ${ }^{1}$ Radiology and ${ }^{2}$ Surgery, University of Michigan Medical Center, Ann Arbor, Michigan, USA, and Departments of ${ }^{3}$ Pathology and ${ }^{4}$ Orthopedic Surgery, Hurley Medical Center, Flint, Michigan, USA
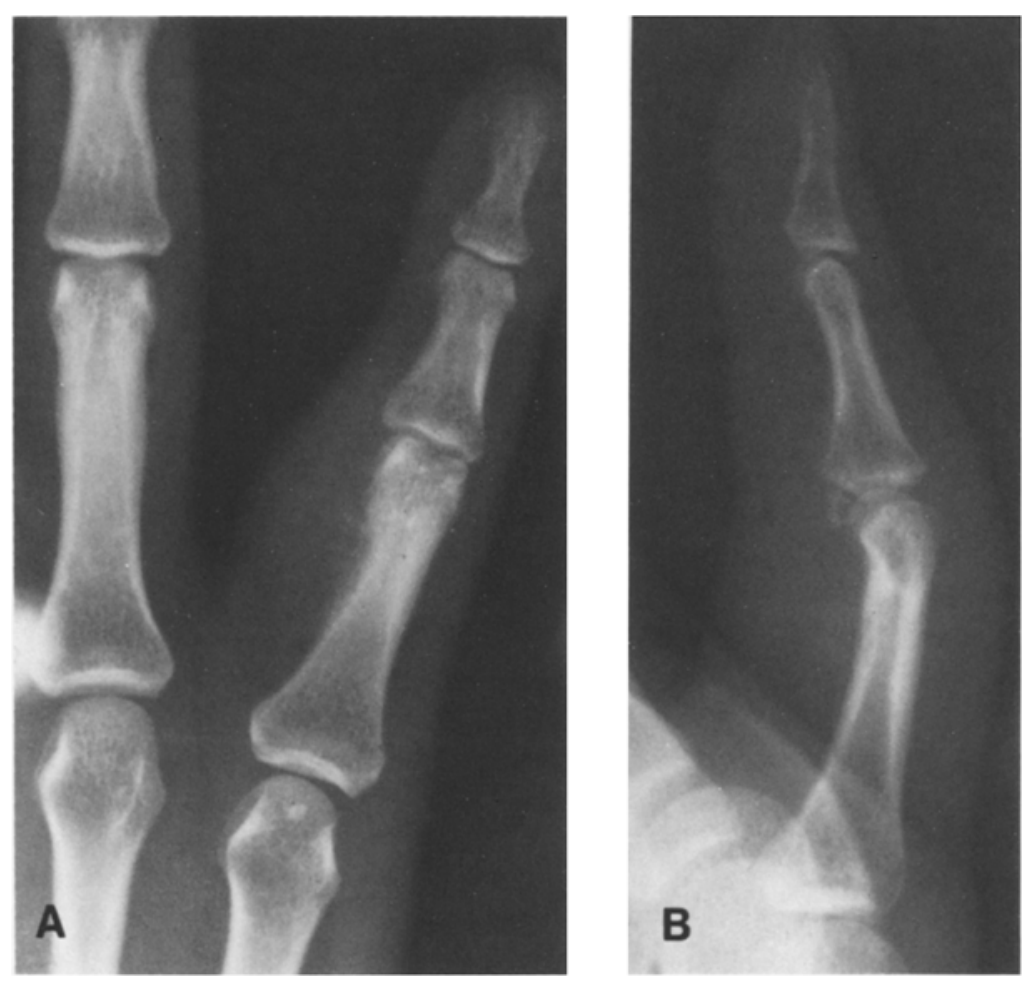

\section{Radiological Studies}

Fig. 1 A, B. Anteroposterior and lateral roentgenograms of the left fifth finger demonstrate faint, irregular calcifications in the soft tissues, just adjacent to the radial aspect of the proximal phalanx of this finger. The cortex of this phalanx is eroded on its radial surface, adjacent to a rather considerable amount of soft tissue swelling; no reactive sclerosis is identified

\section{Clinical Information}

A 22-year-old, right-handed woman presented with a three-month history of painful, progressive swelling around the proximal aspect of the left fifth

\footnotetext{
* Presented by Dr. William Martel at the Annual Meeting of the International Skeletal Society in San Francisco, Califor nia, September 1982
}

Address reprint requests to: William Martel, M.D., Department of Radiology, Box 013, University Hospitals, 1405 E. Ann Street, Ann Arbor, MI 48109, USA finger. Several months prior to the onset of her symptoms she sustained an injury to the proximal interphalangeal joint of the affected finger. Except for acute swelling and discomfort, her recovery from that injury initially had been unremarkable.

On examination, a tender mass was palpated along the radial aspect of the proximal phalanx of the fifth finger, associated with generalized soft tissue swelling.

A biopsy was performed. 


\section{Diagnosis: Florid Reactive Periostitis Proximal Phalanx of the Left 5th Finger}

The differential diagnosis includes principally conventional osteosarcoma, parosteal osteosarcoma, periosteal osteosarcoma, parosteal chondroma, parosteal chondromasarcoma and low-grade osteomyelitis.

The histological sections from the biopsy specimen show a pattern typical of the diagnosis. Varying quantities of osteoid, bone, cartilage and proliferating fibrous tissue are present. In some areas, the fibroblastic proliferation appears dominant and is closely associated with osteoid as well as cartilage. The fibrous component tends to be ac- tive, with large spindled nuclei and prominent nucleoli. Mitoses are present, but are considered to be within the limits of normal. Binucleation of the cellular mitoses is absent. An occasional benign giant cell is observed. The adjacent nodular stroma exhibits a myxoid alteration. A meager inflammatory component, consisting primarily of mononu-
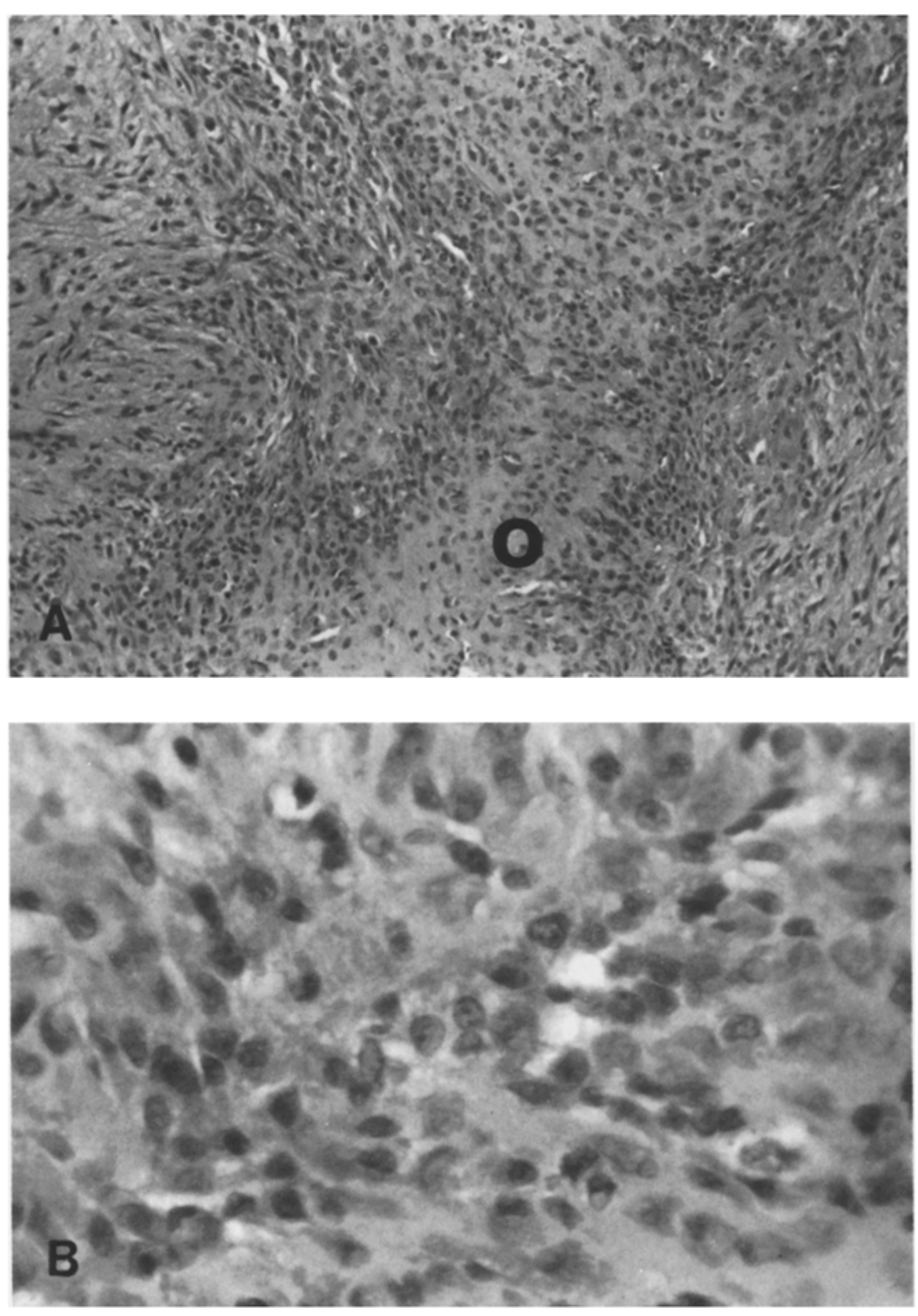

\section{Pathological Studies}

Fig. 2

A This photomicrograph of the biopsy specimen $(\mathrm{H}$ and $\mathrm{E}$ stain $\times 50)$ shows nodularity of the stroma which is reminiscent of nodular fasciitis. Osteoid (O) is surrounded by cellular stroma. The cellularity of the stroma and the osteoid together could be confused with a malignant lesion

B Photomicrograph of the same specimen $(\mathrm{H}$ and $\mathrm{E}$ stain- $\times 100)$ shows that the osteoid present contains many osteocytes and osteoblasts. The overall uniformity of the cells indicates a benign lesion. Scattered mononuclear cells are evident 

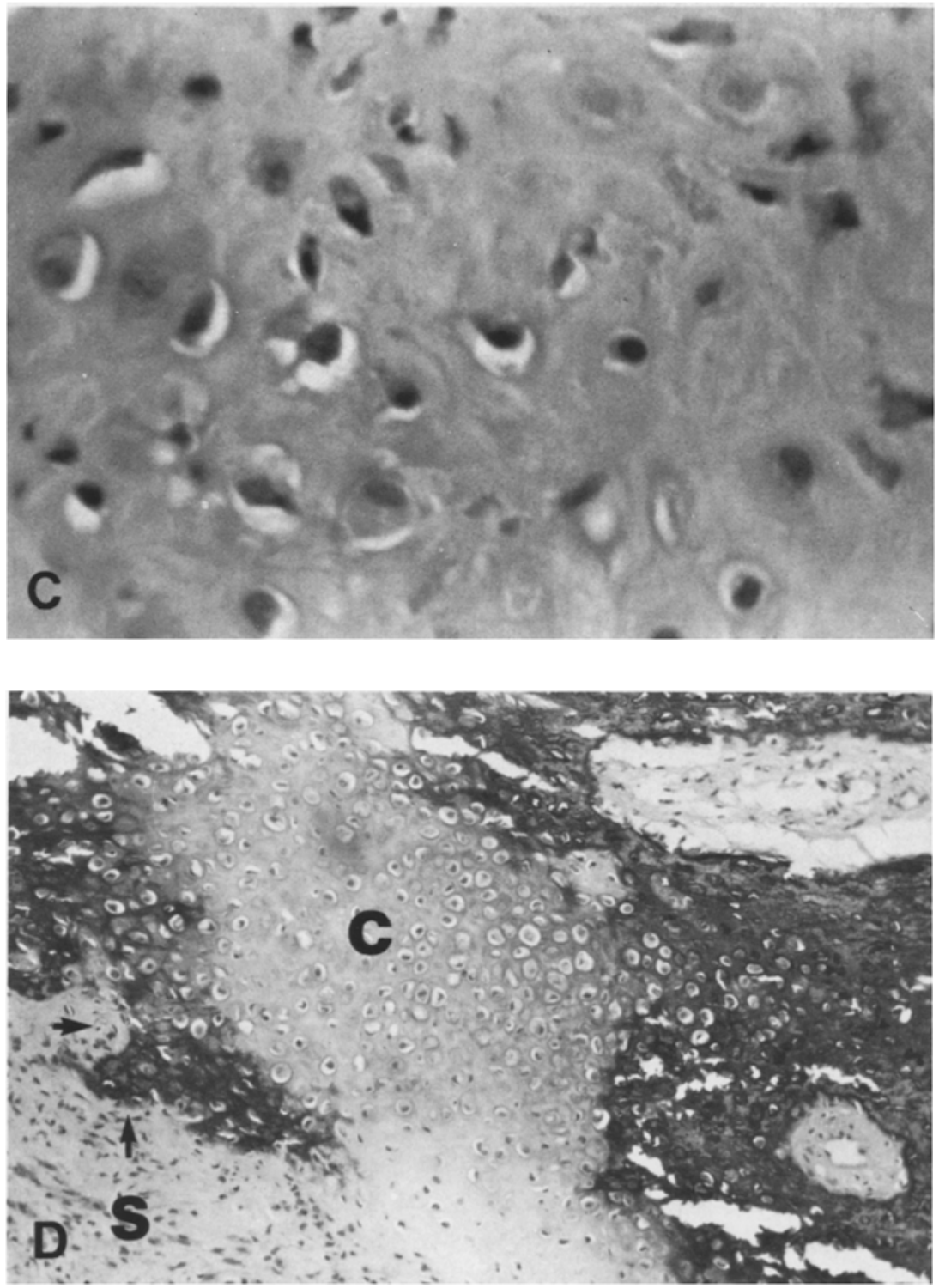

C Photomicrograph of the same biopsy speciment $(\mathrm{H}$ and $\mathrm{E}$ stain $-\times 142.5)$ shows the presence of cellular hyaline cartilage and florid reactive periostitis
D Another photomicrograph, again from the same specimen ( $\mathrm{H}$ and $\mathrm{E}$ stain- $\times 44)$, shows mineralized bone and cartilage (C) in the lesion. The stroma (S) shows a myxoid quality. Osteoclastic resorption (arrows) is noted clear cells and focal extravasation of erythrocytes, is noted in both cellular and myxoid portions of the lesion (Fig. 2A-D).

\section{Discussion}

This reactive lesion of the periosteum or closely adjacent fascia has been termed "florid reactive periostitis" by Spjut and Dorfman [13]. The process typically involves the small bones of the hands or feet in young individuals. The mean age in the series published by Spjut and Dorfman was 22.8 years. Patients usually report a history of progressive, localized swelling over a period of several months. Pain and erythema are present on occa- sion. Approximately half the patients have reported minor trauma to the affected part at sometime prior to the onset of symptoms.

Radiologically, soft tissue swelling virtually is always present, with varying degrees of periosteal reaction which may be manifested as smooth and laminated. On other occasions (presumably in more mature examples of the process), a smooth, solidly mineralized block of the periosteum is identified. As in this case, additional calcification in the soft tissues adjacent to the bone may be present. Spjut and Dorfman reported, but did not illustrate, a small niche in the cortex of their case No. 1. In all other previously reported cases the underly- 
ing cortex has remained intact. The case reported here is unusual in that frank irregularity and erosion of the cortex were present. This particular appearance raised concern for the possibility of malignancy and led to the biopsy.

As indicated previously, the histological pattern was consistant with the diagnosis of florid reactive periostitis.

The similarity between florid reactive periostitis and neoplastic lesions has been noted previously [13]. Hutler et al. [7] used the designation "parosteal fasciitis" to emphasize a close anatomical approximation to bone. They also stressed the resemblence, histologically, to fasciitis occurring in other anatomical regions. McCarthy et al. [10] summarized five cases of parosteal fasciitis involving the bones of the hand, similar to the twelve reported by Spjut and Dorfman. These two authors preferred the term "florid reactive periostitis" to highlight the fact that these lesions are commonly, although not invariably associated with the periosteum.

The similarity to so-called "myositis ossificans" has also been noted [13]. Many authors [1, $4,12]$ have emphasized that myositis ossificans is a poor descriptive term, because skeletal muscle is involved infrequently in such lesions. Dahlin [4] uses a term "heterotopic ossification" instead, and the authors will do the same ${ }^{1}$. Florid reactive periostitis simply may be the specific manifestation in the hand of the more general class of reactive lesions in the soft tissue adjacent to bone.

The lesional tissue of this entity, as well as heterotopic ossification, contains proliferating fibroblasts with mitotic figures but without frank anaplasia. Varying amounts of osteoid, bone and cartilage are present in the histological sections. Furthermore, in all these lesions the ossification is typically most pronounced at the periphery of the mass.

The similarity to heterotopic ossification is significant for another reason. The cortical destruction in the present case was especially distinctive. However, Poznanski [11] noted that myositis ossificans (heterotopic ossification) in the hand may be characterized initially by a soft tissue mass, with subsequent periosteal reaction and rarefaction of the underlying cortex. The cortical erosion in this published case was especially disconcerting. Although primary malignant neoplasms occur rarely in the bones of the hands (and feet), such a possibility could not be completely excluded on the ba-

\footnotetext{
1 Murray and Jacobson use the term "post traumatic mineralization" in instances of "conventional myositis ossificans" (personal communication).
}

sis of the clinical presentation and the radiological studies.

Of the 962 osteosarcomas reported in Dahlin's series of bone tumors [4], only 12 were distal to the wrist or ankle. In such locations, the lesion (osteosarcoma) first presents as a firm, localized, soft tissue swelling, which later becomes painful. Typically, the radiological presentation is unlike that in the present case, in that dense sclerosis of the medullary cavity, with obvious destruction of the cortex and extension of the sclerotic mass into the adjacent soft tissues, usually is present $[2,5$, 11].

Parosteal (or juxtacortical) osteosarcoma is more difficult to differentiate from the benign lesion herein reported. This neoplasm is relatively uncommon, with only 36 cases of the 962 cases of osteosarcoma reported in Dahlin's series. Parosteal osteosarcoma arises on the outer surface of the cortex of an affected bone. At one point the lesion is firmly attached to the cortex and grows in a mushroom fashion, often encircling the bone. The medullary space typically is uninvolved. Although parosteal osteosarcoma has been reported in the hand [11], it is exceedingly rare at this site. This neoplasm is usually distinguishable, radiologically, from florid reactive periostitis in that the malignant tumor is quite sclerotic and broad-based. A thin, lucent line may be observed separating the mass of bone from the cortex in parosteal osteosarcoma. However, in its early stages, the lesion may appear similar to florid reactive periostitis. In 1939, Coley and Higinbotham [3] reported a case of a slowly-growing osteosarcoma involving the proximal phalanx of the fifth finger, which presented radiologically as a thin, faint layer of ossification adjacent to the periosteum. Inasmuch as parosteal osteosarcoma was not described as a distinct entity until 1951 [6], the case reported by Coley and Higinbotham may have represented parosteal osteosarcoma. Mangini [9] presented two cases of parosteal osteosarcoma involving a finger. In one instance, the ossification and swelling of the soft tissue closely resembled the features described in the present reported case of florid reactive periostitis.

With regard to the pathological findings, although, in a few instances florid, fibroblastic proliferation observed early in the course of florid reactive periostitis may make exlusion of malignancy difficult, this entity typically lacks the frankly anaplastic appearance of a sarcoma. In addition, as in heterotopic ossification, florid reactive periostitis may exhibit a "zoning" effect $[1,13]$ with increasing maturity observed in the periphery of the lesion (where ossification also tends to be 
greatest); the central portion of the lesion shows decreased maturity of the cellular pattern. In contrast, sarcomatous lesions typically show decreasing differentiation toward the periphery with the most anaplastic cells at the margins. In addition, in the typical osteosarcoma, the central portion of the lesion is relatively dense radiologically in contrast with a relatively lucent periphery because of a maximum of tumor bone and osteoid comprising the center of the lesion and a tendency for more stromal tissue and less tumor bone and osteoid at the periphery of the lesion.

A periosteal (juxtacortical) chondroma may present as a slowly-growing, painful mass in digit of the hand $[4,8,12]$. Radiologically, these lesions may contain irregular, central calcification.

Typically, however, the underlying cortex appears eroded or even "gouged-out", with a relatively prominent sclerotic reaction, not present in this case of florid reative periostitis. Nests of cartilage cells predominate histologically in the periosteal chondroma.

Infection was not a strong consideration radiologically in this case, nor did any histological features exist to suggest this entity. More destruction of the underlying bone would be expected with most forms of pyogenic osteomyelitis. However, conceivably, a low-grade inflammatory lesion associated with any one of variety of organisms, such as tuberculous dactylitis (now quite rare), could manifest periosteal reaction and overlying soft tissue swelling [11].

In summary, florid reactive periostitis is a metaplastic transformation arising in the periosteum, presenting with localized pain and soft tissue swelling. A history of trauma to the affected area may be obtained. Careful attention to both radiological and histological features of the lesion are impor- tant in order to differentiate it from rare, malignant neoplasms (e.g. osteosarcoma, parosteal osteosarcoma), which may develop in the hands (or feet).

The typical radiological and histological features are discussed and the differential diagnostic possibilities considered (conventional osteosarcoma, parosteal osteosarcoma, periosteal osteosarcoma, parosteal chondroma, parosteal chondrosarcoma, heterotopic ossification and osteomyelitis), with which this entity may be confused.

\section{References}

1. Ackerman LV (1958) Extra-osseous localized non-neoplastic bone and cartilage formation (so-called myositis ossificans). J Bone Joint Surg [Am] 40:279

2. Carroll RE (1957) Osteogenic sarcoma in the hand. J Bone Joint Surg [Am] 39:325

3. Coley BL, Higinbotham NL (1939) Tumor primary in the bones of the hands and feet. Surgery $5: 112$

4. Dahlin D (1978) Bone tumors, 3rd edn. Charles C Thomas, Springfield, pp 28, 226, 376

5. Drompp BW (1961) Bilateral osteosarcoma in the phalanges of the hand. J Bone Joint Surg [Am] 43:199

6. Geschickter CF, Copeland MM (1951) Parosteal osteoma of bone: A new entity. Ann Surg 133:790

7. Hutter RVP, Foote FW, Francis KC, Higinbotham NL (1962) Parosteal fasciitis. Am J Surg 104:800

8. Lichtenstein L (1977) Bone tumors, 5th edn. CV Mosby, Saint Louis, p 392

9. Mangini V (1967) Tumors of the skeleton of the hand. Bull Hosp Joint Dis 28:61

10. McCarthy EF, Ireland DCR, Sprague BL (1976) Parosteal (nodular) fasciitis of the hand. $J$ Bone Joint Surg [Am] $58: 714$

11. Poznanski AK (1974) The hand in radiologic diagnosis. WB Saunders, Philadelphia, pp 420, 437, 391, 430

12. Schajowicz $F$ (1981) Tumors and tumorlike lesions of bone and joints. Springer, New York, pp 115, 490

13. Spjut HJ, Dorfman HD (1981) Florid reactive periostitis of the tubular bones of the hands and feet. Am J Surg Pathol 5:423 